\title{
The compartment bag test (CBT) for enumerating fecal indicator bacteria: Basis for design and interpretation of results
}

\author{
Andrew D. Gronewold ${ }^{\mathrm{a}, \mathrm{b}, *}$, Mark D. Sobsey ${ }^{\mathrm{c}}$, Lanakila McMahan ${ }^{\mathrm{d}}$ \\ a NOAA, Great Lakes Environmental Research Laboratory, Ann Arbor, MI, USA \\ ${ }^{\mathrm{b}}$ Department of Civil and Environmental Engineering, University of Michigan, Ann Arbor, MI, USA \\ ${ }^{c}$ Department of Environmental Sciences and Engineering, Gillings School of Global Public Health, University of North Carolina Chapel Hill, Chapel Hill, NC, USA \\ ${ }^{\mathrm{d}}$ United States Agency for International Development (USAID), Washington, D.C., USA
}

\section{H I G H L I G H T S}

- The statistical basis for the compartment bag test is documented.

- Interpretation of test results reflects methodological uncertainty.

- Bayesian MCMC methods are employed to infer bacteria concentrations.

\section{A R T I C L E I N F O}

\section{Article history:}

Received 11 January 2017

Received in revised form 6 February 2017

Accepted 6 February 2017

Available online $\mathrm{xxxx}$

Editor: D. Barcelo

\section{Keywords:}

Compartment bag test

Water quality

Drinking water

Human health

Statistical methods

\section{G R A P H I C A L A B S T R A C T}

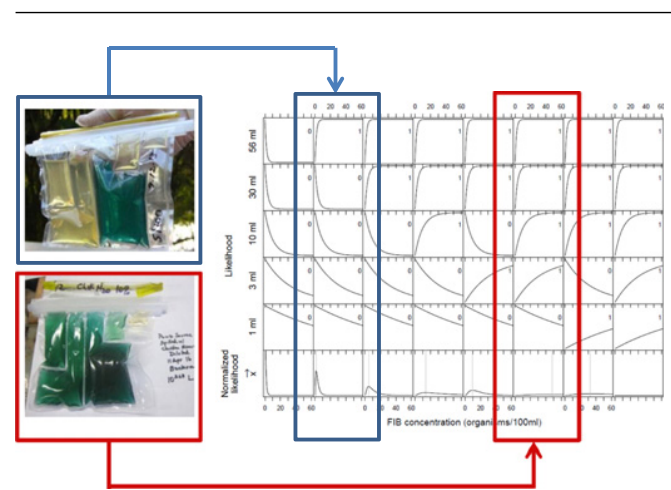

\begin{abstract}
A B S T R A C T
For the past several years, the compartment bag test (CBT) has been employed in water quality monitoring and public health protection around the world. To date, however, the statistical basis for the design and recommended procedures for enumerating fecal indicator bacteria (FIB) concentrations from CBT results have not been formally documented. Here, we provide that documentation following protocols for communicating the evolution of similar water quality testing procedures. We begin with an overview of the statistical theory behind the CBT, followed by a description of how that theory was applied to determine an optimal CBT design. We then provide recommendations for interpreting CBT results, including procedures for estimating quantiles of the FIB concentration probability distribution, and the confidence of compliance with recognized water quality guidelines. We synthesize these values in custom user-oriented 'look-up' tables similar to those developed for other FIB water quality testing methods. Modified versions of our tables are currently distributed commercially as part of the CBT testing kit.
\end{abstract}

Published by Elsevier B.V.

\section{Introduction}

Ensuring readily-available high quality drinking water is fundamental to human health and has important connections to

\footnotetext{
* Corresponding author at: NOAA, Great Lakes Environmental Research Laboratory, Ann Arbor, MI, USA

E-mail address: drew.gronewold@noaa.gov (A.D. Gronewold).
}

socioeconomic status, commercial and industrial growth, and overall quality of life (Mekonnen and Hoekstra, 2016). The challenge of providing that ensurance is met in different ways around the world; in some communities, drinking water supplies are assumed protected if they are adequately separated from wastewater and other sources of contamination (George, 2008). In others, routine water quality testing is used to ensure compliance with recognized standards (Gleick, 1998; Novotny, 2003). Testing kits that support these assessments 
often require a skilled technician to collect, analyze, and interpret results, as well as microbiological laboratory facilities. In regions of the world without these resources and where the time from water withdrawal (from its source) to consumption is short, alternative testing procedures are needed.

To address this gap in global water quality protection, researchers at the University of North Carolina Chapel Hill and Duke University developed a simple kit for enumerating FIB concentrations that is portable, relatively inexpensive, and provides easy-to-interpret results (Stauber et al., 2014). This kit, commonly referred to as the compartment bag test (or CBT), is currently manufactured and distributed by Aquagenx, LLC and has been tested and used in communities around the world (Murcott et al., 2015; Weiss et al., 2016). To date, however, the statistical basis for the design and recommended interpretation of results from the CBT have not been formally documented.

Here, following documentation for the development of similar water quality testing kits (McCrady, 1915; de Man, 1977; Tillett and Coleman, 1985; Haas, 1989; McBride et al., 2003), we begin with an overview of the statistical theory behind the CBT, followed by examples of how that theory was applied to determine an optimal CBT design. We then provide recommendations for interpreting CBT results, including procedures for estimating quantiles of the FIB concentration probability distribution, as well as procedures for calculating the confidence of compliance with World Health Organization (WHO) drinking water quality guidelines (McBride and Ellis, 2001; Borsuk et al., 2002; World Health Organization, 2004). We synthesize these values in custom user-oriented 'look-up' tables similar to those developed for other FIB testing kits (de Man, 1977). Finally, we explore the sensitivity of CBT results to departures from assumptions in the underlying statistical models, and from recommended protocols for sample collection and handling.

\section{Experimental}

\subsection{Statistical basis for interpreting $C B T$ results}

The CBT is a manufactured clear plastic multi-compartment bag into which $100 \mathrm{ml}$ of a water sample is distributed (Stauber et al., 2014). Each compartment contains a growth substrate designed to detect groups of FIB (such as hydrogen sulphide producers), or specific bacteria such as Escherichia coli (EC), by turning a distinctive color (e.g. blue-green) indicating growth of "target" (e.g. FIB or EC) bacteria during an incubation period. The CBT will yield a pattern of 'positive' and 'negative' compartments from which a user can infer the FIB concentration of the original sample following the common assumption (Greenwood and Yule, 1917; Cochran, 1950; Woodward, 1957; El-Shaarawi et al., 1981; Hurley and Roscoe, 1983; de Man, 1983; Haas and Heller, 1988; Woomer et al., 1990; Briones and Reichardt, 1999) that, for a given sample, the number of target bacteria $\left(y_{i}\right)$ in compartment $i(i \in[1, m]$ and $m$ is the total number of compartments) with volume $v_{i}$ (assuming a well-mixed sample) is well-represented by a Poisson probability distribution $y_{i} \sim \operatorname{Po}\left(\lambda_{i}=\right.$ $c v_{i} / 100$ ) with FIB concentration $c$ (in organisms per $100 \mathrm{ml}$ ), and mean and variance $\lambda_{i}$. The probability of a positive compartment of volume $v_{i}$ is $1-\exp \left(-c v_{i} / 100\right)$. The joint probability of any pattern of positive and negative compartments $\vec{x}$ (where the over-arrow superscript denotes a row vector, $x_{i} \in[0,1]$ and $x=1$ indicates a positive compartment) is then expressed as the product of a series of $m$ independent Bernoulli trials:

$f(\vec{x} \mid \vec{v}, c) \propto \prod_{i=1}^{m}\left(1-e^{-c v_{i} / 100}\right)^{x_{i}}\left(e^{-c v_{i} / 100}\right)^{1-x_{i}}$
Conventional interpretations of presence/absence test kits for FIB often focus on a deterministic solution to $c$ from Eq. (1). This value is commonly referred to as the "most probable number" (or MPN) and can be calculated as (Hurley and Roscoe, 1983; McBride, 2005; Gronewold and Wolpert, 2008)

$\operatorname{MPN}=\underset{c}{\operatorname{argmax}}\left[\prod_{i=1}^{m}\left(1-e^{-c v_{i} / 100}\right)^{x_{i}}\left(e^{-c v_{i} / 100}\right)^{1-x_{i}}\right]$

We implement this formulation using the uniroot function in the R statistical software package (R Core Team, 2014). Corresponding code is included in the Supplementary Information.

Multiple methods have been developed for expressing uncertainty in the MPN, however most do not explicitly acknowledge that the probability distribution of the MPN for a given pattern of positive and negative compartments is typically discrete and multimodal, while the probability distribution of the FIB concentration is almost always unimodal and continuous (Klee, 1993; Gronewold and Wolpert, 2008). Therefore, in addition to reporting conventional MPN values, we propose two interpretations of CBT results that allow for a more robust understanding of the uncertainty in the FIB concentration and how that uncertainty affects the confidence of compliance with water quality guidelines (McBride and Ellis, 2001; Gronewold and Borsuk, 2009, 2010). The first is based on calculating quantiles of the likelihood function of the FIB concentration (Eq. (1), written as a function of $c$ for given $\vec{x}$ and $\vec{v}$ ), as well as the probability that the FIB concentration exceeds 1, 10, 100, or 1000 organisms per $100 \mathrm{ml}$.

The second interpretation is based on a Bayesian analysis of CBT results (Bernardo and Ramon, 1998; Press, 2003; Bolstad, 2004) where the posterior probability distribution of the FIB concentration $c$ is proportional to the product of the likelihood function (Eq. (1)) and prior probability distribution $\pi(c)$ :

$f(c \mid \vec{x}, \vec{v}) \propto \pi(c) f(\vec{x} \mid \vec{v}, c)$

One advantage of this approach is that it allows for expression of $a$ priori assumptions about the potential range of the FIB concentration in a water sample. Methods based on the likelihood function alone, in contrast, implicitly assume $a$ priori that FIB concentrations ranging from 0 to $\infty$ are equally likely; an assumption analogous to a belief that gross contamination is just as likely as a FIB concentration within a few orders of magnitude of (or even well below) WHO water quality guidelines. This a priori belief is just one of many a CBT user might have about water quality at a particular sampling location (Press, 2003). Here, we present calculations based on a lognormal prior $\pi(c)=\operatorname{LN}\left(\mu=0, \sigma^{2}=100\right)$, with log-concentration mean $\mu$ and variance $\sigma^{2}$, intended to represent an a priori belief that the FIB concentration is most likely low, but that extreme FIB concentrations are possible. We view further investigation of impacts of alternative priors on CBT results as an important area for future research.

It is informative to note that previous studies have explored alternative probability models for interpreting multiple-compartment water quality analysis results, including the negative binomial model and variations of the Poisson model that account for thinning and dispersion (Christian and Pipes, 1983; El-Shaarawi et al., 1981; Messner and Wolpert, 2002; Crainiceanu et al., 2003). Recent research, however (see Gronewold et al., 2008; Wu et al., 2014), indicates that only extreme and persistent violations of the Poisson probability model would justify application of an alternative probability model.

Finally, following Eq. (1), we calculate the relative likelihood of each possible combination of positive and negative compartments. Results of this calculation provide an indication of CBT outcomes that are most likely, and those that (because they are extremely unlikely) might indicate contamination or thinning of individual 


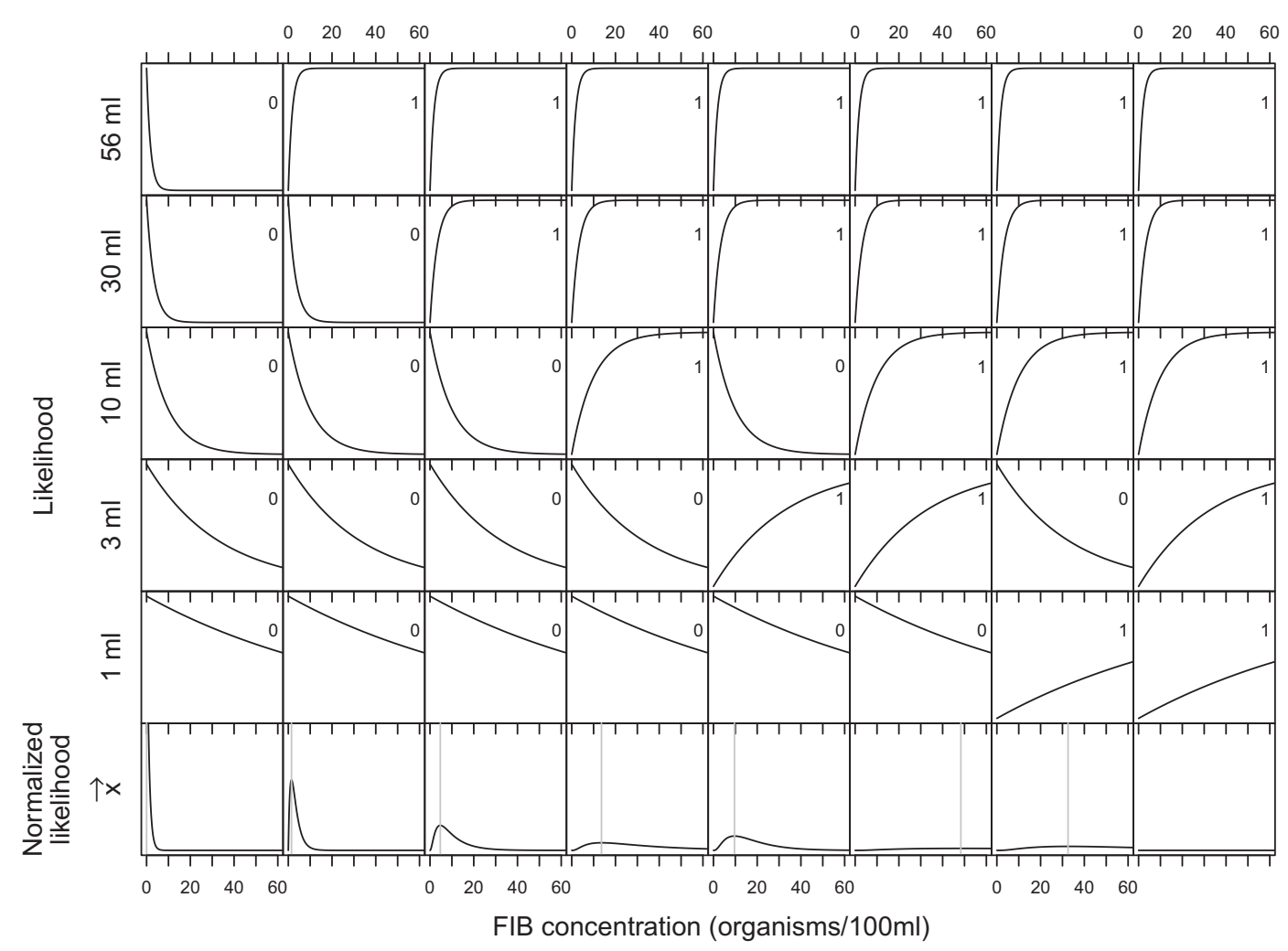

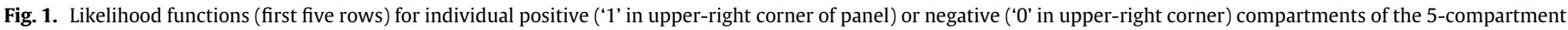

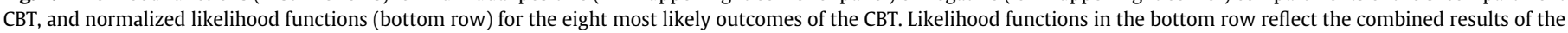

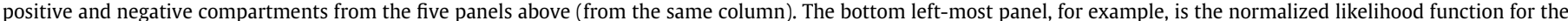

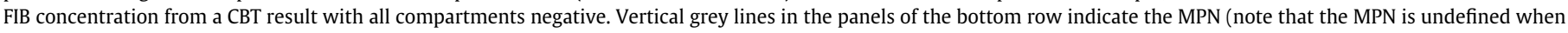
all compartments are positive).

compartments and would therefore warrant additional testing and verification.

\subsection{Design criteria}

The number and volume of compartments of the CBT is based on consideration of a range of criteria including ease of manufacturing, minimization of potential user error (such as unintentionally distributing more or less water into each CBT compartment than intended), and results that are readily translatable into health riskbased metrics. More specifically, the ideal CBT design yields a pattern of positive and negative compartments that are easy to translate into FIB concentrations with uncertainty bounds relevant to human health risks. For most applications of the CBT, we expect these risks will be assessed using FIB concentration numeric limits prescribed in WHO water quality guidelines. We assess compliance with this criteria by inferring FIB concentrations associated with each possible result (i.e. each combination of positive and negative compartments) of a particular CBT design, and then comparing these concentrations to established water quality criteria and standards.

To demonstrate our approach, we provide a comparison between two CBT designs. The first (the design ultimately employed in practice) is a CBT with five compartments with volumes (in ml) $\vec{v}=$ $\{56,30,10,3,1\}$. The second is a CBT with seven compartments with volumes $\vec{v}=\{37,32,16,8,4,2,1\}$. These design options evolved out of a qualitative consideration of the aforementioned criteria, as well as the constraints that the cumulative volume of all compartments equal $100 \mathrm{ml}$, and that the compartment volumes span as broad a range as possible without multiple compartments of the same volume.

Table 1

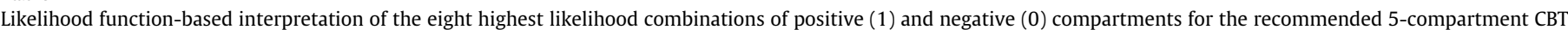

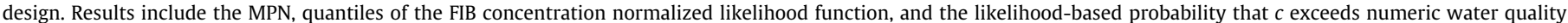

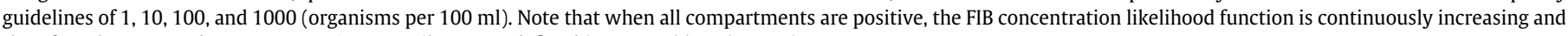
therefore the MPN and FIB concentration quantiles are undefined (Gronewold et al., 2010).

\begin{tabular}{|c|c|c|c|c|c|c|c|c|c|c|c|c|c|c|c|}
\hline \multicolumn{5}{|c|}{ Highest likelihood combinations of positive ( 1 ) and negative ( 0 ) compartments } & \multirow[t]{2}{*}{ MPN } & \multirow[t]{2}{*}{$q_{2.5}$} & \multirow[t]{2}{*}{$q_{5.0}$} & \multirow[t]{2}{*}{$q_{25.0}$} & \multirow[t]{2}{*}{$q_{75.0}$} & \multirow[t]{2}{*}{$q_{95.0}$} & \multirow[t]{2}{*}{$q_{97.5}$} & \multicolumn{4}{|c|}{ Likelihood that $c>$} \\
\hline $56 \mathrm{ml}$ & $30 \mathrm{ml}$ & $10 \mathrm{ml}$ & $3 \mathrm{ml}$ & $1 \mathrm{ml}$ & & & & & & & & 1 & 10 & 100 & 1000 \\
\hline 0 & 0 & 0 & 0 & 0 & 0.0 & $<0.1$ & $<0.1$ & 0.3 & 1.4 & 3.0 & 3.7 & 0.33 & 0.00 & 0.00 & 0.00 \\
\hline 1 & 0 & 0 & 0 & 0 & 1.5 & 0.4 & 0.5 & 1.5 & 4.4 & 8.1 & 9.7 & 0.85 & 0.02 & 0.00 & 0.00 \\
\hline 1 & 1 & 0 & 0 & 0 & 4.7 & 1.5 & 2.1 & 4.8 & 13.2 & 24.8 & 29.7 & 0.99 & 0.39 & 0.00 & 0.00 \\
\hline 1 & 1 & 1 & 0 & 0 & 13.6 & 4.8 & 6.4 & 15.1 & 44.1 & 84.4 & 101.8 & 1.00 & 0.87 & 0.03 & 0.00 \\
\hline 1 & 1 & 0 & 1 & 0 & 9.6 & 3.3 & 4.3 & 9.2 & 23.0 & 39.7 & 46.5 & 1.00 & 0.71 & 0.00 & 0.00 \\
\hline 1 & 1 & 1 & 1 & 0 & 48.3 & 16.4 & 22.4 & 55.5 & 170.0 & 331.0 & 400.5 & 1.00 & 0.99 & 0.50 & 0.00 \\
\hline 1 & 1 & 1 & 0 & 1 & 32.6 & 10.9 & 14.3 & 31.6 & 81.4 & 141.8 & 166.5 & 1.00 & 0.98 & 0.16 & 0.00 \\
\hline 1 & 1 & 1 & 1 & 1 & NA & NA & NA & NA & NA & NA & NA & NA & NA & NA & NA \\
\hline
\end{tabular}




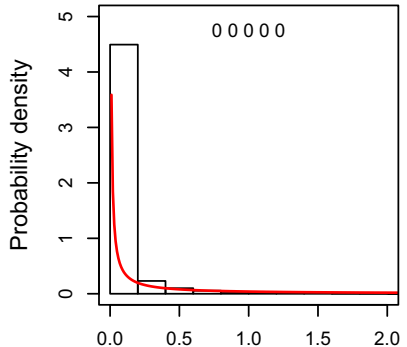

FIB concentration (org/100mL)

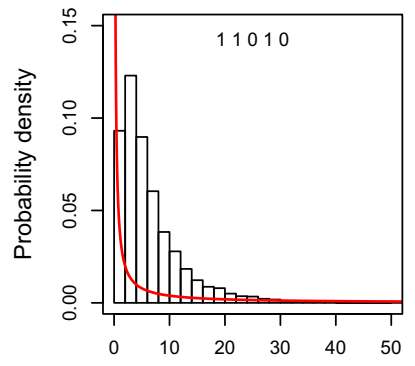

FIB concentration (org/100mL)

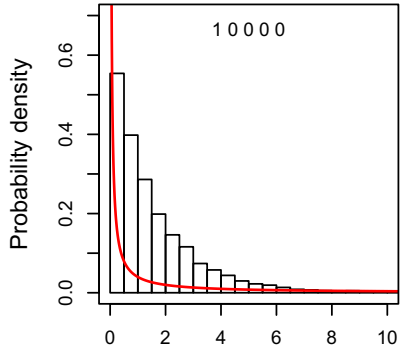

FIB concentration (org/100mL)

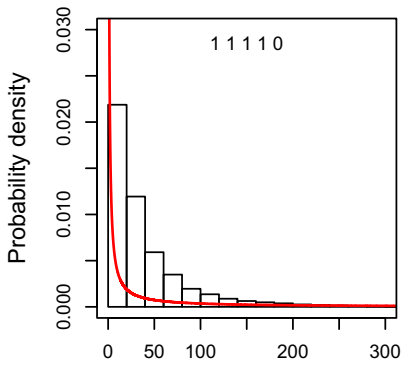

FIB concentration (org/100mL)

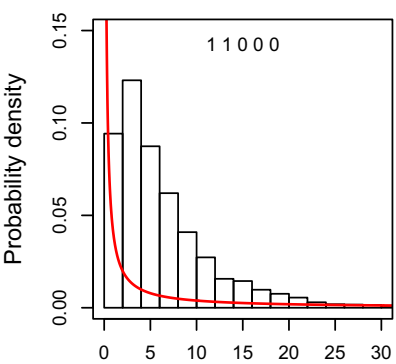

FIB concentration (org/100mL)

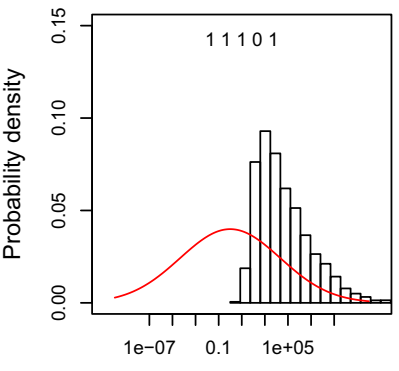

FIB concentration (org/ $100 \mathrm{~mL})$

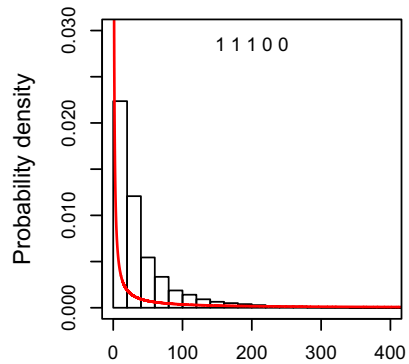

FIB concentration (org/100mL)

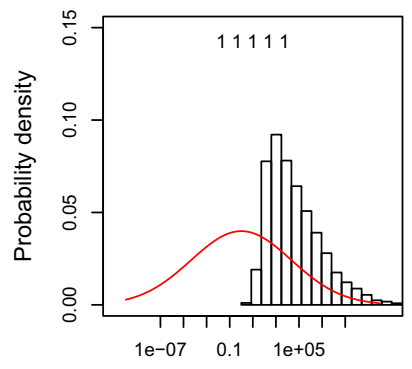

FIB concentration (org/100mL)

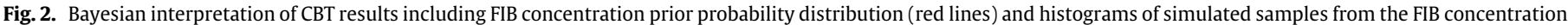

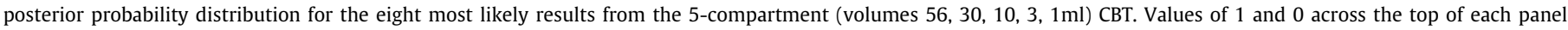

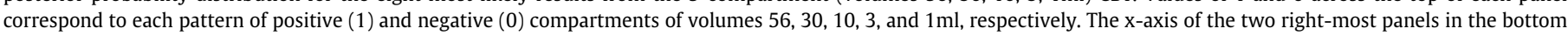
row is plotted on a logarithmic scale for clarity.

For each of the two test designs, we first calculated the full FIB concentration likelihood function for each possible CBT result, and then implemented our Bayesian interpretation by simulating samples from the posterior probability distribution of the FIB concentration (Eq. (3)) for each possible CBT result using Markov chain Monte Carlo (MCMC) procedures in the software program WinBUGS (Lunn et al., 2000). We ran each MCMC chain until it reached convergence, indicated by a potential scale reduction factor $\hat{R}$ (Gelman et al., 2004) close to 1.0. WinBUGS code used to simulate the posterior probability distribution for $c$ for the $\vec{v}=\{56,30,10,3,1\}$ CBT design is included in the Supplementary Information. From the likelihood functions and posterior probability distributions, we calculate a series of quantiles, as well as the likelihood (or posterior probability) that the FIB concentration exceeds $1,10,100$, or 1000 organisms per $100 \mathrm{ml}$.

\subsection{Sensitivity analysis}

To better understand the sensitivity of CBT results to potential variations in user handling (including violations of the assumptions in our statistical models), we repeat the simulation described in the previous section for the 5-compartment CBT using hypothetical compartment volumes (in $\mathrm{ml}$ ) of $\vec{v}=\{58.4,30.5,14.5,2.5,0.7\}$ and $\vec{v}=\{32.3,33.5,23.3,4.9,3.4\}$. These volume sequences were obtained from an informal (unpublished) study by one of the authors at the University of North Carolina - Chapel Hill in which roughly twenty individuals with a range of $\mathrm{CBT}$ experience used the $\mathrm{CBT}$, and the actual water sample volumes they distributed into each compartment were recorded. The two selected sequences represent, respectively, moderate and severe departures from the intended 5-compartment CBT design with compartment volumes $\vec{v}=\{56,30,10,3,1\}$.

\section{Results and discussion}

Of the 32 potential combinations of positive and negative compartments for the 5-compartment CBT, we find that there are appreciable differences in the relative likelihood of each outcome (see Table S1 in the Supplementary Information). Some results (particularly those for which the $56 \mathrm{ml}$ compartment is positive) are

Table 2

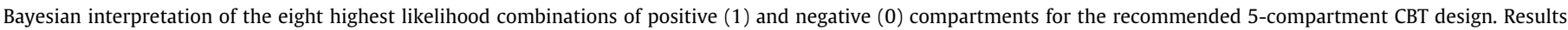

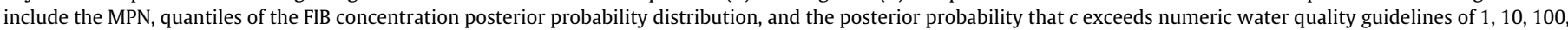

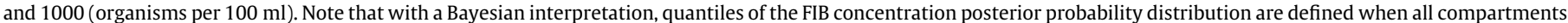

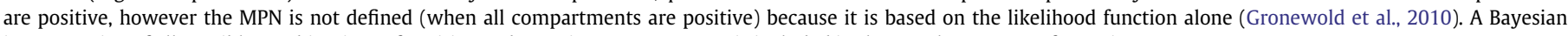
interpretation of all possible combinations of positive and negative compartments is included in the Supplementary Information.

\begin{tabular}{|c|c|c|c|c|c|c|c|c|c|c|c|c|c|c|c|}
\hline \multicolumn{5}{|c|}{ Highest likelihood combinations of positive ( 1 ) and negative ( 0 ) compartments } & \multirow[t]{2}{*}{ MPN } & \multirow[t]{2}{*}{$q_{2.5}$} & \multirow[t]{2}{*}{$q_{5.0}$} & \multirow[t]{2}{*}{$q_{25.0}$} & \multirow[t]{2}{*}{$q_{75.0}$} & \multirow[t]{2}{*}{$q_{95.0}$} & \multirow[t]{2}{*}{$q_{97.5}$} & \multicolumn{4}{|c|}{ Post. prob. that $c>$} \\
\hline $56 \mathrm{ml}$ & $30 \mathrm{ml}$ & $10 \mathrm{ml}$ & $3 \mathrm{ml}$ & $1 \mathrm{ml}$ & & & & & & & & 1 & 10 & 100 & 1000 \\
\hline 0 & 0 & 0 & 0 & 0 & 0 & $<0.1$ & $<0.1$ & $<0.1$ & $<0.1$ & 0.5 & 0.8 & 0.02 & 0.00 & 0.00 & 0.00 \\
\hline 1 & 0 & 0 & 0 & 0 & 1.5 & $<0.1$ & 0.1 & 0.4 & 2.2 & 4.9 & 6.2 & 0.51 & 0.00 & 0.00 & 0.00 \\
\hline 1 & 1 & 0 & 0 & 0 & 4.7 & 0.6 & 0.9 & 2.5 & 8.3 & 18.1 & 22.0 & 0.94 & 0.18 & 0.00 & 0.00 \\
\hline 1 & 1 & 1 & 0 & 0 & 13.6 & 2.8 & 3.8 & 11.1 & 49.7 & 142.5 & 189.3 & 1.00 & 0.78 & 0.10 & 0.00 \\
\hline 1 & 1 & 0 & 1 & 0 & 9.6 & 0.6 & 0.8 & 2.5 & 8.3 & 18.9 & 23.6 & 0.94 & 0.19 & 0.00 & 0.00 \\
\hline 1 & 1 & 1 & 1 & 0 & 48.3 & 2.8 & 3.9 & 11.0 & 50.8 & 143.6 & 191.2 & 1.00 & 0.78 & 0.10 & 0.00 \\
\hline 1 & 1 & 1 & 0 & 1 & 32.6 & 37.4 & 70.7 & 734.7 & $8.5 \times 10^{5}$ & $1.2 \times 10^{9}$ & $1.9 \times 10^{10}$ & 1.00 & 1.00 & 0.92 & 0.70 \\
\hline 1 & 1 & 1 & 1 & 1 & NA & 36.9 & 67.2 & 702.9 & $8.5 \times 10^{5}$ & $1.4 \times 10^{9}$ & $2.0 \times 10^{10}$ & 1.00 & 1.00 & 0.93 & 0.73 \\
\hline
\end{tabular}


Table 3

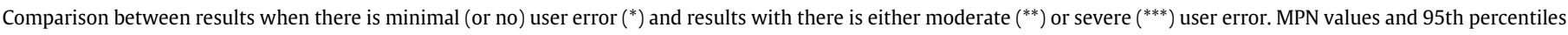

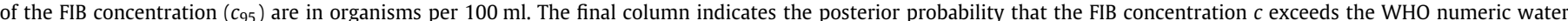

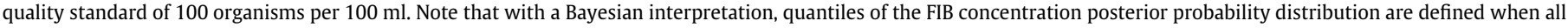

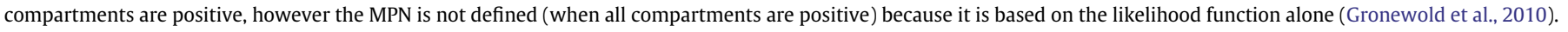

\begin{tabular}{|c|c|c|c|c|c|c|c|c|c|c|c|c|c|}
\hline \multicolumn{5}{|c|}{ Highest likelihood combinations of pos. (1) and neg. (0) compartments } & \multicolumn{3}{|c|}{ MPN } & \multicolumn{3}{|c|}{$c_{95}$} & \multicolumn{3}{|c|}{$\mathrm{P}(c>100)$} \\
\hline $56 \mathrm{ml}$ & $30 \mathrm{ml}$ & $10 \mathrm{ml}$ & $3 \mathrm{ml}$ & $1 \mathrm{ml}$ & * & ** & *** & $*$ & ** & $* * *$ & * & ** & $* * *$ \\
\hline 0 & 0 & 0 & 0 & 0 & 0.0 & 0.0 & 0.0 & 0.5 & 0.5 & 0.4 & 0.0 & 0.0 & 0.0 \\
\hline 1 & 0 & 0 & 0 & 0 & 1.5 & 1.4 & 1.2 & 4.9 & 4.6 & 4.1 & 0.0 & 0.0 & 0.0 \\
\hline 1 & 1 & 0 & 0 & 0 & 4.7 & 4.1 & 3.4 & 18.1 & 14.0 & 10.2 & 0.0 & 0.0 & 0.0 \\
\hline 1 & 1 & 1 & 0 & 0 & 13.6 & 13.0 & 8.4 & 142.5 & 175.0 & 50.2 & 0.1 & 0.1 & 0.0 \\
\hline 1 & 1 & 0 & 1 & 0 & 9.6 & 7.8 & 5.8 & 18.9 & 14.4 & 10.2 & 0.0 & 0.0 & 0.0 \\
\hline 1 & 1 & 1 & 1 & 0 & 48.3 & 60.9 & 19.6 & 143.6 & 178.8 & 49.5 & 0.1 & 0.1 & 0.0 \\
\hline 1 & 1 & 1 & 0 & 1 & 32.6 & 36.2 & 17.3 & $1.2 \times 10^{9}$ & $1.8 \times 10^{9}$ & $0.9 \times 10^{9}$ & 0.9 & 0.9 & 0.8 \\
\hline 1 & 1 & 1 & 1 & 1 & NA & NA & NA & $1.4 \times 10^{9}$ & $1.8 \times 10^{9}$ & $1.0 \times 10^{9}$ & 0.9 & 0.9 & 0.8 \\
\hline
\end{tabular}

quite likely while others are highly improbable. This is an important distinction because highly unlikely CBT outcomes might indicate one or more potential problems with sample handling and analysis (including thinning or contamination of a particular compartment) and warrant additional investigation. To underscore this point, and to simplify our discussion of alternative CBT interpretations, we hereafter focus on results from only the eight most likely outcomes of the CBT.

FIB concentration likelihood functions reflecting information content of individual CBT compartments (top five rows Fig. 1), and of each combination of positive and negative compartments for the eight most likely CBT results (bottom row Fig. 1), provide insight into origins of uncertainty in CBT-based water quality assessments (see also Table 1). For example, a CBT result with a pattern of positive (1) and negative (0) compartments (with volumes 56, 30, 10,3, and $1 \mathrm{ml}$ ) of $\vec{x}=\{1,1,0,1,0\}$ has an MPN of 9.6 (organisms per $100 \mathrm{ml}$ ) with moderate certainty in the FIB concentration. A CBT result for which the pattern of positive and negative compartments is $\vec{x}=$ $\{1,1,1,1,0\}$ has a higher MPN (48.3) and more uncertainty in the FIB concentration because of the difference in the information content of the $10 \mathrm{ml}$ compartment. A positive $10 \mathrm{ml}$ compartment (by itself) indicates that the FIB concentration is almost certainly above roughly 40 organisms per $100 \mathrm{ml}$, while a negative $10 \mathrm{ml}$ compartment indicates that the concentration is almost certainly below 40 organisms per $100 \mathrm{ml}$. The contrast between the information in these two results underscores not only the relative value of keeping the CBT simple (by minimizing the number of compartments, for example)

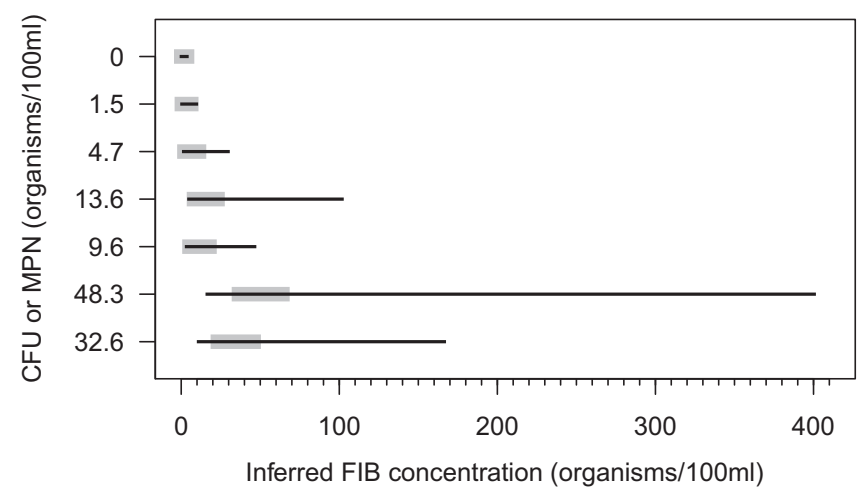

Fig. 3. FIB concentration $95 \%$ likelihood intervals based on seven of the eight most likely results of the 5-compartment CBT (intervals for a CBT result with all compartments positive are not shown because the likelihood function is continuously increasing and the MPN is undefined). Thin black segments represent likelihood intervals derived from the CBT. Thick grey segments represent likelihood intervals derived from conventional membrane filtration (MF) analyses with CFU values that correspond to MPN values from the CBT. The top-most pair of segments, for example, includes FIB concentration 95\% likelihood intervals from (thin black segment) a CBT result with an MPN of 0 , and (thick grey segment) an MF result with a CFU of 0 . and easy to implement, but also the potential sensitivity of CBT outcomes to variations in sample handling.

A Bayesian interpretation of results from the 5-compartment CBT with $\vec{v}=\{56,30,10,3,1\}$ (Fig. 2 and Table 2) indicates how explicit quantification of a priori beliefs about the FIB concentration in a sample can propagate into different perceptions of human health risk (Fig. 2) when compared to interpretations based on the likelihood function alone, particularly for CBT outcomes with an intrinsically broad likelihood function (e.g. a result of $\vec{x}=\{1,1,1,1,0\}$ ). In areas where there is a long history of high quality drinking water, for example, a prior probability distribution reflecting a strong belief in a relatively low FIB concentration may be helpful in guiding water use management decisions when there is insufficient information content in the likelihood function alone.

We also find that the 5-compartment CBT design (Tables 1 and 2) provides a robust basis for distinguishing samples based on compliance with WHO water quality guidelines, particularly when compared to our alternative design with seven compartments (see Table S2 in the Supplementary Information). For nearly all of the most likely results of the 5-compartment CBT, we can make a relatively confident statement about the range of the sample FIB concentration, and about compliance with each numeric limit in the WHO guidelines. This statement may depend, as we have shown, on whether a likelihood or Bayesian interpretation is used. In either case, a probabilistic interpretation enhances information from conventional MPN values alone; water quality experts are often comfortable with MPN values, but not with quantifying associated uncertainties when the MPN is derived from a novel and unconventional testing kit such as the CBT.

Our assessment of the potential impacts of user error (Table 3 and Supplementary Information) suggests that the 5-compartment CBT test is relatively robust to both moderate and severe errors. More specifically, we find that moderate handling errors would not have changed the perceived probability of violating the WHO water quality guideline of 100 organisms per $100 \mathrm{ml}$ (a value indicating 'very high risk' water). Furthermore, we find that severe errors, while leading to a slightly lower perceived probability of violating the WHO water quality guideline of 100 organisms per $100 \mathrm{ml}$, would also have been very unlikely to lead to a different perception of risk than what would have been inferred had there been no error.

Finally, we acknowledge that users of the CBT have inquired about the uncertainty in CBT results relative to uncertainties in more conventional water quality testing tools, including (for example) membrane filtration (MF) tests (Dufour and Cabelli, 1975; Dufour et al., 1981; El-Shaarawi et al., 1981). A comparison between the 95\% likelihood intervals from our analysis of the CBT (Table 1) and 95\% likelihood intervals from MF tests with colony-forming unit (CFU) values matching MPN values from the CBT (Gronewold and Wolpert, 2008) indicates that (Fig. 3), for very low (i.e. less than 5 organisms per $100 \mathrm{ml}$ ) FIB concentrations, the confidence intervals 
are quite similar and that the differences are more extreme for FIB concentration close to and above 10 (organisms per $100 \mathrm{ml}$ ). A Bayesian interpretation of CBT results (Table 2) could affect the range of these intervals and might in fact be desirable should water quality management officials (and other CBT users) find that the likelihood-based intervals do not provide enough informative at higher concentrations. We suggest investigation of impacts of alternative prior distributions on inferred FIB concentration uncertainty and compliance with WHO water quality guidelines as a high priority for future research.

\section{Acknowledgments}

The authors thank Craig Stow for comments on early versions of this manuscript, as well as Song Qian and James Crooks for helpful discussions on Bayesian inference. This is NOAA-GLERL contribution number 1849.

\section{Appendix A. Supplementary information}

Supplementary data to this article can be found online at http:// dx.doi.org/10.1016/j.scitotenv.2017.02.055.

\section{References}

Bernardo, J.M., Ramon, J.M., 1998. An introduction to Bayesian reference analysis: inference on the ratio of multinomial parameters. 47 (1), 101-135.

Bolstad, W.M., 2004. Introduction to Bayesian Statistics. Wiley-Interscience, Hoboken, N.J.

Borsuk, M.E., Stow, C.A., Reckhow, K.H., 2002. Predicting the frequency of water quality standard violations: a probabilistic approach for TMDL development. Environ. Sci. Technol. 36 (10), 2109-2115.

Briones, A.M., Reichardt, W., 1999. Estimating microbial population counts by 'most probable number' using Microsoft Excel. J. Microbiol. Methods 35 (2), 157-161.

Christian, R.R., Pipes, W.O., 1983. Frequency distributions of coliforms in water distribution systems. Appl. Environ. Microbiol. 45 (2), 603-609.

Cochran, W.G., 1950. Estimation of bacterial densities by means of the 'most probable number'. Biometrics 6 (2), 105-116.

Crainiceanu, C.M., Stedinger, J.R., Ruppert, D., Behr, C.T., 2003. Modeling the U.S. national distribution of waterborne pathogen concentrations with application to Cryptosporidium parvum. Water Resour. Res. 39 (9).

de Man, J.C., 1977. MPN Tables for more than one test. Eur. J. Appl. Microbiol. Biotechnol. 4 (4), 307-316.

de Man, J.C., 1983. MPN tables, corrected. Eur. J. Appl. Microbiol. Biotechnol. 17 (5), 301-305.

Dufour, A.P., Cabelli, V.J., 1975. Membrane-filter procedure for enumerating component genera of coliform group in seawater. Appl. Microbiol. 29 (6), 826-833.

Dufour, A.P., Strickland, E.R., Cabelli, V.J., 1981. Membrane-filter method for enumerating Escherichia coli. Appl. Environ. Microbiol. 41 (5), 1152-1158.

El-Shaarawi, A.H., Esterby, S.R., Dutka, B.J., 1981. Bacterial density in water determined by Poisson or negative binomial distributions. Appl. Environ. Microbiol. 41 (1), 107-116

Gelman, A.J., Carlin, J.B., Stern, H.S., Rubin, D.B., 2004. Bayesian Data Analysis. Chapman \& Hall/CRC, Boca Raton, Florida.

George, R., 2008. The Big Necessity. Metropolitan, New York.

Gleick, P.H., 1998. Water in crisis: paths to sustainable water use. Ecol. Appl. 8 (3), 571-579.
Greenwood, M., Yule, G.U., 1917. On the statistical interpretation of some bacteriological methods employed in water analysis. J. Hyg. 16 (1), 36-54.

Gronewold, A.D., Borsuk, M.E., 2009. A software tool for translating deterministic model results into probabilistic assessments of water quality standard compliance. Environ. Model Softw. 24 (10), 1257-1262.

Gronewold, A.D., Borsuk, M.E., 2010. Improving water quality assessments through a hierarchical Bayesian analysis of variability. Environ. Sci. Technol. 44 (20), 7858-7864.

Gronewold, A.D., Borsuk, M.E., Wolpert, R.L., Reckhow, K.H., 2008. An assessment of fecal indicator bacteria-based water quality standards. Environ. Sci. Technol. 42 (13), 4676-4682.

Gronewold, A.D., Myers, L., Swall, J.L., Noble, R.T., 2010. Addressing uncertainty in fecal indicator bacteria dark inactivation rates. Water Res. 45 (2), 652-664.

Gronewold, A.D., Wolpert, R.L., 2008. Modeling the relationship between most probable number (MPN) and colony-forming unit (CFU) estimates of fecal coliform concentration. Water Res. 42 (13), 3327-3334.

Haas, C.N., 1989. Estimation of microbial densities from dilution count experiments Appl. Environ. Microbiol. 55 (8), 1934-1942.

Haas, C.N., Heller, B., 1988. Test of the validity of the Poisson assumption for analysis of most-probable-number results. Appl. Environ. Microbiol. 54 (12), 2996-3002.

Hurley, M.A., Roscoe, M.E., 1983. Automated statistical analysis of microbial enumeration by dilution series. J. Appl. Bacteriol. 55 (1), 159-164.

Klee, A.J., 1993. A computer program for the determination of most probable number and its confidence limits. J. Microbiol. Methods 18 (2), 91-98.

Lunn, D.J., Thomas, A., Best, N., Spiegelhalter, D., 2000. WinBUGS-a Bayesian modelling framework: concepts, structure, and extensibility. Stat. Comput. 10 (4), 325-337.

McBride, G.B., 2005. Using Statistical Methods for Water Quality Management. Issues, Problems and Solutions. John Wiley \& Sons Ltd, Chichester, UK.

McBride, G.B., Ellis, J.C., 2001. Confidence of compliance: a Bayesian approach for percentile standards. Water Res. 35 (5), 1117-1124.

McBride, G.B., McWhirter, J.L., Dalgety, M.H., 2003. Uncertainty in most probable number calculations for microbiological assays. J. AOAC Int. 86 (5), 1084-1088.

McCrady, M.H., 1915. The numerical interpretation of fermentation tube results. J. Infect. Dis. 17 (1), 183-212.

Mekonnen, M.M., Hoekstra, A.Y., 2016. Four billion people facing severe water scarcity. Sci. Adv. 2 (2). e1500323.

Messner, M.J., Wolpert, R.L., 2002. Cryptosporidium and Giardia occurrence in ICR drinking water sources - statistical analysis of ICR data. In: McGuire, M.J. (Ed.), Information Collection Rule Data Analysis. American Water Works Association Research Foundation, Denver, CO, pp. 463-481.

Murcott, S., Keegan, M., Hanson, A., Jain, A., Knutson, J., Liu, S., Tanphanich, J. Wong, T.K., 2015. Evaluation of microbial water quality tests for humanitarian emergency and development settings. Procedia Eng. 107, 237-246.

Novotny, V., 2003. Water Quality: Diffuse Pollution and Watershed Management. 2nd ed., John Wiley \& Sons, New Jersey.

Press, S.J., 2003. Subjective and Objective Bayesian Statistics: Principles, Models, and Applications. Wiley-Interscience, Hoboken, NJ

Core Team, R., 2014. R: a language and environment for statistical computing. Vienna, Austria. http://www.r-project.org.

Stauber, C., Miller, C., Cantrell, B., Kroell, K., 2014. Evaluation of the compartment bag test for the detection of Escherichia coli in water. J. Microbiol. Methods 99, 66-70.

Tillett, H.E., Coleman, R., 1985. Estimated numbers of bacteria in samples from non-homogeneous bodies of water - how should MPN and membrane filtration results be reported? J. Appl. Bacteriol. 59 (4), 381-388.

Weiss, P., Aw, T.G., Urquhart, G.R., Galeano, M.R., Rose, J.B., 2016. Well water quality in rural Nicaragua using a low-cost bacterial test and microbial source tracking. J. Water Health 14 (2), 199-207.

Woodward, R.L., 1957. How probable is the most probable number? J. Am. Water Works Assoc. 49 (1), 1060-1068

Woomer, P.L., Bennett, J., Yost, R., 1990. Overcoming the inflexibility of most-probable-number procedures. Agron. J. 82 (2), 349-353.

World Health Organization, 2004. Guidelines for drinking-water quality: recommendations. Tech. Rep., Geneva

Wu, J., Gronewold, A.D., Rodriguez, R.A., Stewart, J., Sobsey, M.D., 2014. Integrating quantitative PCR and Bayesian statistics in quantifying human adenoviruses in small volumes of source water. Sci. Total. Environ. 470, 255-262. 